\title{
Knowledge Helps, but Alone It Is Not Enough
}

\author{
Murray F. Brennan, MD \\ Department of Surgery, Memorial Sloan-Kettering Cancer Center, New York, NY, USA
}

\section{To the Editor:}

The understanding of soft tissue sarcoma has progressed impressively in the last two decades. Delineation of the biology, molecular diagnosis, and outcome have been carefully characterized with defined prognostic indicators. The challenge has been to identify if biology can predict behavior; that has now been clearly established. More importantly, can biology and predictive outcome mandate treatment change, and finally can treatment, based on biology and known outcome, be altered to reflect improved outcome?

Dr Pollock and his coauthors examine this question by defining retroperitoneal liposarcoma into well-differentiated (WD) and dedifferentiated (DD) subtypes. ${ }^{1}$ They examined 135 patients over the last 10 years at their institution. This significant experience in a major sarcoma center allows important observations. As expected, WD was mostly primary and DD was mostly recurrent. Chemotherapy was rarely used in WD, and more aggressive resection was required in DD, while a third to half of all patients required multivisceral resection. The initial complete gross resection was obtained in both groups, and, as expected, recurrence was greater in the dedifferentiated group. As previously identified, metastatic disease is rare in well-differentiated liposarcoma and local recurrence can be late and prolonged.

While the title of the present manuscript, "Two Different Diseases Possibly Requiring Different Treatment Approaches," is most enticing, the data

Published online March 18, 2008.

Address correspondence and reprint requests to: Murray F. Brennan, MD; E-mail: brennanm@mskcc.org

Published by Springer Science+Business Media, LLC $\odot 2008$ The Society of Surgical Oncology, Inc. predominantly confirm that accurate diagnosis of histological subtype predicts recurrence. A recent nomogram based on 800 patients with liposarcoma has confirmed the importance of histological subtype. ${ }^{1}$ Few would challenge the authors questioning of whether, with such different outcomes, treatment should be different; however, how different is different? The real challenge, alluded to in the title, is what "different" treatment should be applied to these "different" diseases. The authors are clearly aware of the different outcomes, and that has already affected management. In both groups radiation is minimally used $(<20 \%)$, whereas chemotherapy is essentially never used in the well-differentiated type and used in the dedifferentiated type in the majority $(73 \%)$. However, the benefits of such different approaches are unclear. Despite aggressive use of multimodality therapy, outcome remains poor with 5 -year survival in DD at $37 \%$. This is similar to that reported by Dalal et al. at $39 \%{ }^{2}$

Unfortunately, the real challenge remains; we can increasingly improve the accuracy of histological diagnosis, molecular characterization, and prediction of outcome, but we are far less sanguine about improvements in treatment that translate into better survival. Importantly, the reverse is true. We are now aware that we can diminish the aggressivity of surgical approaches when the biology is favorable and the consequence of recurrence limited. The authors confirm that less aggressive surgical approaches for WD are appropriate. The presence of WD liposarcoma especially an asymptomatic locally recurrent lesion does not mandate operation! It is fortunate that our knowledge increases and unfortunate that we cannot transfer that increase of knowledge into more effective therapy. We take some consolation in the fact that we can decrease morbidity of surgical therapy by better understanding of biology. 


\section{REFERENCES}

1. Lahat G, Anaya DA, Wang X, et al. Resectable well differentiated versus dedifferentiated liposarcomas: two different diseases possibly requiring different treatment approaches. Ann Surg Oncol 2008; 15 (DOI: 10.1245/s10434-007-9805-x).
2. Dalal KM, Kattan MW, Antonescu CR, et al. Subtype specific prognostic nomogram for patients with primary liposarcoma of the retroperitoneum, extremity, or trunk. Ann Surg 2006; 244:381-91. 